\title{
A Study on the Development of Mobile Based SNS-UCC Writing Tool
}

\author{
Hae Sun No ${ }^{1}$ and Dae Woong Rhee ${ }^{2}$ \\ ${ }^{1}$ Ph.D. Course, Dept of Game, Graduate School, \\ SangMyung University, 7 HongJi-Dong Jongno-Gu, Seoul, Korea \\ hs $4 \mathrm{k} 2$ @naver.com \\ ${ }^{2}$ Division of Digital Media, College of Computer Software \& Digital Media, \\ SangMyung University 7 HongJi-Dong Jongno-Gu, Seoul, Korea \\ rhee219@smu.ac.kr
}

\begin{abstract}
Driven by the development of wireless services with the advent of smart phone along with iPhone, wireless services are drawing attentions rather than PC internet based wired services. Since this technique is a mobile device judged as being in an environment suitable to provide SNS, there also need be researches. This research proposed mobile based SNS-UCC design through mobile based SNS-UCC writing tool development cases.
\end{abstract}

Keywords: SNS, UCC, Mobile, Writing Tool, Development.

\section{Introduction}

Presently throughout the world, interactive services that offer amusements to users and are represented by UCC and Web 2.0, the SNS based UCC services, are extensively developed. The background SNS and UCC grew thus active is due to the desires of users to produce contents personally and consume them. As they prefer information in forms that they can understand intuitively, the types of contents change from image to moving pictures.[1] Another reason SNS and UCC grew active is that the opportunities to use wireless network increased owing to supply of $3 \mathrm{G}$ phone and high performance terminal devices like smart phone etc showed up.[2]

\section{Development of Mobile Based SNS-UCC Writing Tool}

SNS based UCC services are produced based on PC internet. However PC internet based SNS-UCC service has the following disadvantages:

The first is the inconvenience of saving personal information. In case of PC internet base, users are faced with the inconvenience that they have to sign up individually at each and every site that they intend to use, and enter and save information on themselves each time.

The second is time and space limitation. PC internet based SNS requires a space that offers PC and internet. In other words, if PC is not available, or if internet cannot be accessed though PC is available, SNS cannot be used. 
The third is the many time and efforts needed in learning the using method. PC internet based UCC writing tool requires users to personally edit story structure or screen setting as well as moving picture, photo, speech and various effects. Therefore, many hours and efforts are required in learning and using such functions.

In developing mobile based SNS-UCC, the following designing principles should assumed as fundamental:

First, easy manipulation and access should be considered in priority. Existing UCC writing tools require many hours and efforts in learning functions to use, so easy access was hard to be attained. Therefore, even beginner should be able to use it easily by making the interface for manipulation easy.

Second, consideration should be given to produce interactive contents that users can use in various fields. Mobile based SNS-UCC writing tool should be developed to be utilized in various fields like UCG(User Created Game), relay novel, music video and advertisement etc as well as existing UCC. The more fields the tool can be utilized in, the more users will use it, and the more diverse types of profit models will be created.

Third, mobile based SNS-UCC supports easy transfer using MMS(Multimedia Message Service) and mobile web. MMS and mobile web should be supported simultaneously by considering the internet environment of touch phone and smart phone that are drawing attention recently as well as existing cellular phone and finally, convenience should be improved through interlinking with PC internet.

Fourth, mobile based SNS-UCC should be designed so that it can be used in any mobile device through compatibility with various platforms. Mobile based SNS-UCC should be able to use writing tool in various mobile devices like smart phone etc represented by iPhone and Android phone as well as the cellular phone that people carry the most.

\section{Cases of Mobile Based SNS-UCC Writing Tool Development}

Mobile based SNS-UCC writing tool is under development through support by 2009 IT Excellent Technology Supporting Project by applying the principle of mobile based SNS-UCC writing tool designing. "SNUC" the mobile based SNS-UCC writing tool is under development by reflecting the 4 designing principles that we went over above, and has the following characteristics:

1) Contents can be produced "anytime anywhere".

In existing UCC production, space that accommodates use of PC was required, thus facing limitation of time and space. However, "SNUC" is of mobile base of easy portability, thus offering the advantage of being able to be produced with no limitation of time or space.

2) Transfer of "emotion" through story telling is possible.

Existing UCC was used often in editing moving picture or photo. "SNUC" can make theme story by utilizing the photo, moving picture or music etc of its own in mobile device, so it can create more emotional story. Also, "SNUC" should be made so that it can produce contents with various story with the conception that people remember contents with story longer. "SNUC" supports production of contents with story by offering structure of story with themes by the situation called theme template. 
3) SNS that can be shared with "anyone".

In the case of existing SNS, information of its own should be entered and saved individually by accessing the site through PC internet. But, mobile device already contains all the information of my own, no entering of information is required. In addition, mobile device is also a tool most widely used in communicating with and establishing relationship with people.

4) Can produce contents "easily and simply".

To produce UCC in the past, developers should learn and use complicated tool, thus requiring long hours in learning the tool and facing hardship in access. However, "SNUC" is considerate enough to accommodate completion of contents only through providing of template in a completed form with themes by the situations and simple modification.

5) Offers the extensibility "that can be used in various fields".

"SNUC" is designed to be used in various fields like novel, film, music video, advertisement, marketing and drama etc as well as the function of SNS-UCC. Owing to the function of storytelling, "SNUC" can be applied in various types like UCG(User Created Game), UMC(User Modified Contents" and URC(User Recreated Contents) etc in addition to UCC.

\section{Conclusion}

Yet, the researches and developments of mobile based SNS and UCC are at beginning stage compared with PC based internet. However, many experts and companies are conducting research and development with significant interest in SNS and UCC.

It is anticipated that once mobile based service with environment suitable for the supply of SNS is presented, not only SNS field but also UCC and Web 2.0 service on such basis will also evolve one step further. In such sense, it is important to establish the principles for the development and designing of mobile based SNS-UCC writing tool.

Development of "SNUC" will play the role of uplifting the reachability of users as writing tool that can be used for easy and simple production in mobile based SNS market which is still at beginning stage.

\section{References}

1. Ahn, S.-H., Song, S.-M.: Status and Development Prospect of UCC Service. Korea Contents Society General Academic Convention Journal 5, 691-697 (2007)

2. Kim, S.-Y.: Trend and Prospect of Mobile SNS Service. Korea Communication Society Journal 26, 19-25 (2009) 